\title{
Reacquisition of learned taste aversions
}

\author{
SAM REVUSKY and SHANNON COOMBES \\ Memorial University of Newfoundland, St. John's, Newfoundland AIC 5S7, Canada
}

\begin{abstract}
In four experiments, it was found that rats subjected to extinction of conditioned taste aversions readily reacquired these aversions when subjected to conditioning once again. Two of the present procedures were very similar to those used by Danguir and Nicolaides in unsuccessful attempts to obtain reconditioning of extinguished taste aversions.
\end{abstract}

In the chemical aversion treatment of alcoholism, therapeutic alcohol aversions are produced by making patients sick after they consume alcoholic beverages (Boland, Mellor, \& Revusky, 1978; Lemere \& Voegtlin, 1950, Voegtlin, Lemere, \& Broz, 1940; Wiens, Montague, Manaugh, \& English, 1976). Because this treatment is similar to the conditioning of flavor aversions in the animal laboratory, some animal experimentation has been concerned with validating techniques of chemical aversion therapy (Elkins, 1974; Revusky, 1973; Revusky \& Gorry, 1973; Revusky, Parker, Coombes, \& Coombes, 1976; Revusky \& Taukulis, 1975; Revusky, Taukulis, Parker, \& Coombes, 1979).

A recent rat experiment suggests that an important feature of present alcohol aversion programs may not be of therapeutic value. This is the use of booster treatments (reconditioning sessions) for patients who have relapsed and extinguished their alcohol aversions. Danguir and Nicolaides (1977) report that if a learned flavor aversion is extinguished, a later pairing of consumption of the flavored solution with sickness will not reinstate the aversion. If so, and if this result is applicable to human alcoholics, booster treatments are useless.

\section{EXPERIMENT 1}

We began by trying to delineate the effect more exactly. Danguir and Nicolaides (1977) reported that extinction of an aversion to saccharin solution interferes markedly with later reconditioning of the aversion to saccharin. Here we tried to find out if extinction of a learned aversion to vinegar solution also would make it difficult to condition an aversion to saccharin solution. This would suggest that extinction of an aversion to any flavor would interfere with conditioning of an aversion to a different flavor.

We thank Michael Lavin for carefully reading and criticizing this manuscript. This work was supported by a grant from the National Research Council of Canada. Reprints may be obtained from the authors at the Department of Psychology, Memorial University of Newfoundland, St. John's, Newfoundland A1C 5S7, Canada.
Danguir and Nicolaides reconditioned aversions only to the previously extinguished flavor, and hence did not consider this question.

Table 1 outlines the procedures for the two focal experimental treatments. The "Ext-tox" treatment corresponds in outline to the original DanguirNicolaides treatment. The rats are conditioned to have a saccharin aversion in Phase 5 after a history in which a saccharin aversion is conditioned (Phase 2) and extinguished (Phase 3). The "Fam-tox" procedure also involves conditioning of a saccharin aversion in Phase 5 , but these animals have a history in which an aversion to vinegar solution was conditioned (Phase 2) and extinguished (Phase 3). Phases 1 and 4 in Table 1 were added to make the two procedures similar in respect to potential extraneous factors. The purpose of Phase 1 was to insure that the saccharin test solution would be just as familiar to the rats subjected to the Fam-tox treatment as to those subjected to the Ext-tox treatment. The purpose of Phase 4 was to insure that, at the time of reconditioning in Phase 5, Group Fam-tox would have had as much recent experience with saccharin as Group Ext-tox. Familiarity of a flavor and the recency of this familiarity are important factors in flavor aversion learning (Farley, McLaurin, Scarborough, \& Rawlings, 1964) and hence should be made similar for the two groups. Of course, because Phases 1 and 4 were added to the Fam-tox procedure, corresponding phases were added to the Ext-tox procedure.

In addition to the Ext-tox and Fam-tox treatments shown in Table 1, there were two similar treatments in which the roles of the vinegar and saccharin flavored solutions were interchanged. Finally, for each of these four groups, there was a control group subjected to the same procedures except that the saccharin or vinegar solution was not paired with toxicosis in Phase 5; it was simply presented alone to yield a baseline preference for the test solution.

\section{Subjects}

\section{Method}

The 84 male Sprague-Dawley rats ranged in ad-lib weight from 189 to $226 \mathrm{~g}$ just before the experiment. They were never removed from their steel wire home cages except for weighings 
Table 1

The Two Focal Treatments of Experiment 1

\begin{tabular}{lll}
\hline & \multicolumn{2}{c}{ Solution } \\
\cline { 2 - 3 } Phases & \multicolumn{1}{c}{ Ext-Tox } & \multicolumn{1}{c}{ Treatments } \\
\hline Phase 1: Familiarization & Vinegar Alone & Saccharin Alone \\
Phase 2: Conditioning & Saccharin-Toxic Pairing & Vinegar-Toxic Pairing \\
Phase 3: Extinction & Saccharin Alone & Vinegar Alone \\
Phase 4: Recency Control & Saccharin Alone, Vinegar Alone & Saccharin Alone, Vinegar Alone \\
Phase 5: Reconditioning & Saccharin-Toxic Pairings & Saccharin-Toxic Pairings \\
\hline
\end{tabular}

Note-Reference to a flavor means the rats had an opportunity to drink a solution with that flavor and "Toxic" means that a toxic injection followed consumption of the indicated flavor.

and injections and had continual access to dry Purina chow and rationed access to fluids as indicated below.

There were two Ext-tox groups of 14 rats each; one was subjected to the procedures outlined in Table 1 and the other was subjected to a version of these procedures in which the roles of saccharin and vinegar solutions were interchanged. Similarly, there were two Fam-tox groups of 14 rats each. There were also two Ext-cont and two Fam-cont groups of 7 rats each; these were treated exactly like the Ext-tox and Fam-tox groups except that they were not injected with lithium in Phase 5.

\section{Procedure}

Phase 1: Familiarization (Days 1-10). Half of the rats were allowed $15 \mathrm{~min}$ daily access to $.4 \%$ (weight/volume) of sodium saccharin solution, and the other half were allowed similar access to $4.0 \%$ (volume/volume) of Heinz-brand cider vinegar. Prior to the first session on this regimen, the rats had been deprived of fluids for 3 days.

Phase 2: Conditioning (Day 11). All rats were given $15 \mathrm{~min}$ access to saccharin or vinegar solution, whichever had not been supplied in Phase 1. As the bottle was removed, each rat was injected intraperitoneally with $1.0 \mathrm{ml}$ of $2.0 \%(\mathrm{w} / \mathrm{v})$ lithium chloride solution. In terms of amount of $\mathrm{LiCl}$ per kilogram, this dose is $30 \%$ lower than that used by Danguir and Nicolaides (1977). Our dose also differs from their dose in that it is markedly hypertonic (.47 molar); according to Nachman and Ashe (1973), hypertonicity does not affect aversive efficacy noticeably.

Phase 3: Extinction (Days 12-21). Each rat received $15 \mathrm{~min}$ daily access to the same flavored solution it had received in Phase 2. On Days 14 and 15 , if a rat did not drink $6 \mathrm{~g}$ in $15 \mathrm{~min}$, it was given successive 15-min drinking sessions until it drank $6 \mathrm{~g}$. Thereafter, all rats drank at least this much.

Phase 4: Recency control (Days 22-25). On each day, each rat was given a 4-min saccharin drinking session and a 4-min vinegar drinking session. These sessions were separated by 1-2 h. On Days 22 and 25 , and saccharin session was administered first. On Days 23 and 24, the vinegar session was first.

Phase 5: Reconditioning and testing (Days 26-34). This consisted of three 3-day cycles. During the first 2 days of each cycle, the rats drank unflavored water for $15 \mathrm{~min}$. On the $3 \mathrm{rd}$ day, the rats drank either saccharin or vinegar solution; in Fam-tox and Famcont groups, this was the same as the solution consumed in Phase 1, while in Ext-tox and Ext-cont groups, it was the same as the solution consumed during Phases 2 and 3. After the first two exposures to this flavored solution (on Days 28 and 31), the rats in Ext-tox and Fam-tox groups were injected with $1.0 \mathrm{ml}$ of $2.0 \%$ $\mathrm{LiCl}$, while the rats in the Ext-cont and Fam-cont groups were not injected.

\section{Results}

Figure 1 shows mean consumption of the flavored solution for each group during the reconditioning and testing in Phase 5 for each day during which the flavored solution was presented. The left side shows the data for rats subjected to saccharin solution in Phase 5. Thus, the Ext-tox curve on the left side is for rats which had been previously conditioned and extinguished on the same saccharin solution used in reconditioning, while the Fam-tox curve is for rats previously conditioned and extinguished on the different vinegar solution. The Ext-cont and Fam-cont curves are for control groups treated just like the Exttox and Fam-tox groups except that the lithium injection was omitted on the two reconditioning days, 28 and 31. The right side of Figure 1 shows corresponding results for the groups subjected to vinegar solution during the reconditioning and testing of Phase 5.

Day 28 was the first reconditioning day, and hence, on that day, consumption of the flavored solution was not yet affected by the reconditioning process. It is apparent that on this day all groups presented with the same flavored solution drank similar amounts. On subsequent days, each of the Fam-tox and Ext-tox groups declined in its fluid consumption; for each of these four groups, the decline from Day 28 to Day 31 was significant at $p<.005$ by a paired

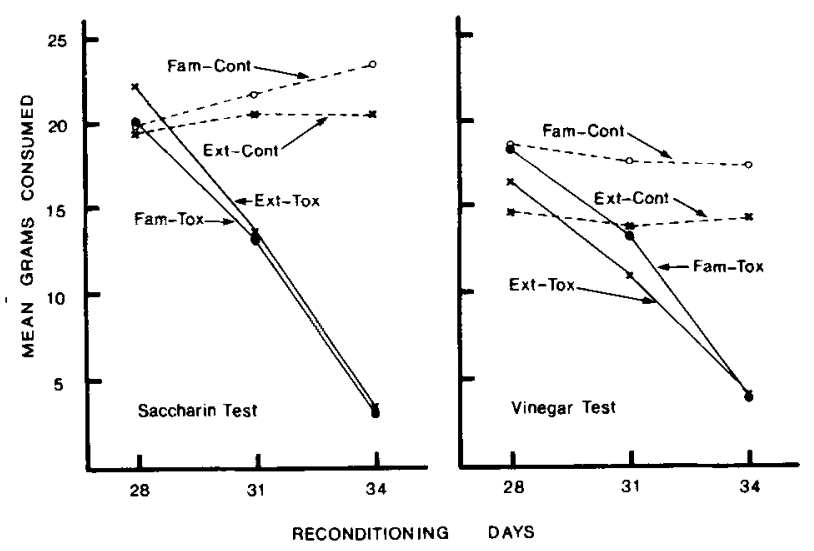

Figure 1. Phase 5 of Experiment 1. Conditioning and testing of aversions to saccharin solution (left side) and to vinegar solution (right side). The Ext-tox groups were previously conditioned and extinguished on the same flavor used in Phase 5, while the Famtox groups were previously conditioned and extinguished with a different flavor. The Ext-cont and Fain-cont groups were controls to test for preference in the absence of aversion training. 
$t$ test and the further decline from Day 31 to Day 34 was significant at $p<.001$. No such decline was apparent among the four control groups.

\section{Discussion}

Not only did the Ext-tox procedure, which contained what seemed to be the essentials of the procedure of Danguir and Nicolaides (1977), fail to prevent reconditioning of flavor aversions, it did not even seem to attenuate the reconditioning when compared with the Fam-tox procedure. In fairness, however, it must be emphasized that the Ext-tox procedure was not an exact replication of the Danguir-Nicolaides procedure.

\section{EXPERIMENT 2}

Experiment 1 showed that rats with a history of a single acquisition and extinction of a flavor aversion can reacquire an aversion to the same flavor. Experiment 2 was mainly concerned with whether rats with a history of two acquisitions and extinctions of the same flavor aversion can acquire a new aversion to that flavor. Since the Ext-tox rats of Experiment 1 already had a history of extinction of a learned flavor aversion and reacquisition of an aversion to the same flavor, it was only necessary to extinguish these animals once more and then recondition them for a third time in order to answer this question.

Table 2 outlines the procedures which the Ext-tox rats of Experiment 1 were subjected to in Experiment 2. In Phase 1, all rats were again subjected to extinction of the flavor aversion which had been conditioned at the end of Experiment 1. In Phase 2, which corresponded to Phase 4 of Experiment 1, all rats were given recent experience both with the previously aversive flavored solution and with the other flavor, which had been made familiar in Experiment 1. In Phase 3 , the rats were divided into subgroups. Five rats subjected to each aversive flavor in Experiment 1 were reconditioned to have an aversion to the same flavor; this treatment corresponds to the Exttox treatment of Experiment 1. Five rats subjected to each aversive flavor in Experiment 1 were conditioned to have an aversion to the opposite flavor; this is a Fam-tox treatment. Finally, for each of the toxicosis groups, there was a group of two rats each treated in exactly the same way except that toxicosis did not follow consumption of the flavored solution in Phase 3 so that conditioned taste aversions would not develop; these formed Ext-cont and Fam-cont groups.

The rats subjected to the Fam-tox treatment in Experiment 1 were discarded. However, the rats subjected to the Fam-cont and Ext-cont treatments in Experiment 1 were used. It will be recalled that all of these rats had a history in which a flavored solution was made aversive on a single occasion and then this aversion was extinguished. We treated all such control rats given an aversion to vinegar in Phase 1 of Experiment 1 as one group and all those given an aversion to saccharin as a second group. We treated these two groups of 14 rats each in exactly the same way we treated the corresponding Ext-tox groups of Experiment 1 as shown in Table 2. Essentially, these rats constituted a replication of Experiment 1 under conditions in which extinction of the previously learned flavor aversion was even more thorough. (We pooled rats subjected to Ext-cont and to Fam-cont procedures in Experiment 1 because the difference in these procedures was small. A later check of the data confirmed that this difference was unimportant.)

\section{Method}

In unspecified details, the method was similar to that of Experiment 1 .

\section{Subjects}

At the end of Experiment 1, there were no reliable differences among the mean body weights of the different categories of rats to be used in Experiment $2[\mathrm{~F}(3,52)=2.01, \mathrm{p}>.10]$. The overall mean weight was $294.5 \mathrm{~g}$.

\section{Procedure}

Phase 1: Extinction (Days 35-44, where Day 34 was the last day of Experiment 1). On Day 35, while the rats were 1 day thirsty, they were given 4 days of continuous access to whichever solution had been paired with toxicosis in Experiment 1. On Day 39, the bottle of flavored solution was removed, and on Day 41, there began 4 days during which the rats received the same fla-

Table 2

Procedure in Experiment 2 for the Ext-Tox Rats and the Control Rats of Experiment 1

\begin{tabular}{|c|c|c|c|c|c|}
\hline $\begin{array}{c}\text { Aversive } \\
\text { Flavor of } \\
\text { Experiment } 1 \\
\end{array}$ & $\begin{array}{l}\text { Phase 1: } \\
\text { Extinction }\end{array}$ & $\begin{array}{c}\text { Phase 2: } \\
\text { Recency Control }\end{array}$ & \multicolumn{2}{|c|}{$\begin{array}{c}\text { Phase } 3 \text { : } \\
\text { Reconditioning }\end{array}$} & $\begin{array}{l}\text { Name of } \\
\text { Treatment }\end{array}$ \\
\hline Saccharin & Saccharin Alone & Saccharin Alone and Vinegar Alone & $\begin{array}{l}\text { Saccharin-Toxic } \\
\text { Vinegar-Toxic } \\
\text { Saccharin Alone } \\
\text { Vinegar Alone }\end{array}$ & $\begin{array}{l}(n=5) \\
(n=5) \\
(n=2) \\
(n=2)\end{array}$ & $\begin{array}{l}\text { Ext-Tox } \\
\text { Fam-Tox } \\
\text { Ext-Cont } \\
\text { Fam-Cont }\end{array}$ \\
\hline Vinegar & Vinegar Alone & Saccharin Alone and Vinegar Alone & $\begin{array}{l}\text { Vinegar-Toxic } \\
\text { Saccharin-Toxic } \\
\text { Vinegar Alone } \\
\text { Saccharin Alone }\end{array}$ & $\begin{array}{l}(\mathrm{n}=5) \\
(\mathrm{n}=5) \\
(\mathrm{n}=2) \\
(\mathrm{n}=2)\end{array}$ & $\begin{array}{l}\text { Ext-Tox } \\
\text { Fam-Tox } \\
\text { Ext-Cont } \\
\text { Fam-Cont }\end{array}$ \\
\hline
\end{tabular}


vored solution for $15 \mathrm{~min}$ per day. On Day 42 , the rats were weighed.

Phase 2: Control for recency (Days 45-49). Vinegar and saccharin solutions were each available for $4 \mathrm{~min}$ per day under the same conditions as in Phase 4 of Experiment 1.

Phase 3: Reconditioning and testing (Days 49-64). The rats were allowed $15 \mathrm{~min}$ daily access to the flavored solution indicated in Table 2 on Days $49,52,54,56,58,60,62$, and 64 ; on the intervening days, they were allowed $15 \mathrm{~min}$ access to unflavored water. On Days 49, 52, and 54, the rats subjected to the Ext-tox and Fam-tox treatments were injected with $1.2 \mathrm{ml}$ of $2.0 \% \mathrm{LiCl}$ solution as the bottle was removed.

\section{Results}

Contrary to what one might expect on the basis of the report by Danguir and Nicolaides (1977), no group of rats failed to develop flavor aversions as a result of ingestion-contingent lithium toxicosis. Figure 2 shows the mean consumption of the flavored solution during the 3 days the rats in Ext-tox and Fam-tox groups were subjected to toxicosis training (Days 49, 52, and 54), as well as consumption during the five extinction sessions (Days 56, 58, 60, 62, and 64). Each quadrant in Figure 2 compares Ext-tox, Fam-tox, Ext-cont, and Fam-cont treatments under a different pair of conditions based upon a 2 by 2 combination of two factors: (1) whether the test solution was saccharin (top row of Figure 2) or vinegar (bottom row), and (2) whether the rats had a history of one prior extinction of a flavor aversion (Ext-1, left side of Figure 2) or of two such extinctions (Ext-2, right side of Figure 2). This arrangement differs from that of Table 2 in that the Ext-tox and Ext-cont treatments outlined in the top half of Table 2 are in the same quadrant as the Fam-tox and Fam-cont treatments outlined in the bottom half of Table 2, and vice versa. (If this change were not made in Figure 2, groups tested on different flavored solution would be compared and this would be scientifically meaningless).

Consumptions among the Ext-tox and Fam-tox groups on Day 49, before there was any effect of the paired lithium injection, were close to those of their appropriate controls. Each of the eight experimental groups then exhibited a significant drop in consumption from Day 49 to Day 52 and another significant drop from Day 52 to Day 54 (.05-level $t$ tests, one tail). There is no sign of a similar drop among any of the eight Ext-cont and Fam-cont groups.

In one respect, however, Figure 2 seems to supply a small amount of support for the notion that prior acquisition and extinction of a saccharin aversion interferes with reacquisition of the saccharin aversion. In the top row of Figure 2, when saccharin was the test solution, the Ext-tox curves are higher than the Fam-tox curves. To determine whether this trend was statistically reliable, we totalled the amounts consumed on Days 52, 54, 56, 58, 60, 62, and 64 for all Ext-tox and Fam-tox rats and subjected the results

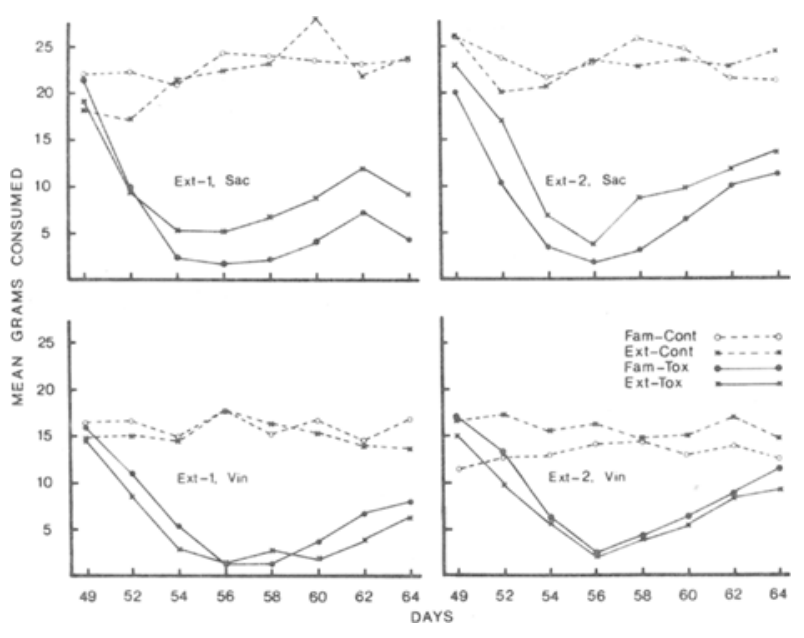

Figure 2. Preferences in Experiment 2 for a test solution among rats subjected to Ext-tox, Fam-tox, Ext-cont, and Fam-cont treatments. Ext-1 refers to rats which had previously been subjected to one conditioning and extinction (left two quadrants); Ext-2 refers to rats which had previously been subjected to two conditionings and extinctions (right quadrants). The top two quadrants refer to the rats tested with saccharin solution and the bottom two quadrants to rats tested with vinegar solution.

to a 2 by 2 by 2 ANOVA. The factors were (1) Exttox vs. Fam-tox, (2) saccharin vs. vinegar test solutions, and (3) Ext-1 vs. Ext-2. The only result of this ANOVA which yielded significance at the .05 level was the interaction between the first two of these factors $[\mathrm{F}(1,32)=4.79, \mathrm{p}<.05]$. The method of orthogonal polynomials showed that among the rats subjected to the saccharin test solution, the Ext-tox procedure produced higher consumptions than the Fam-tox procedure $[F(1,32)=4.70, p<.05]$; there was no similar effect among the rats subjected to vinegar solution in Figure $2(\mathrm{~F}<1.0)$.

The finding that the Ext-tox procedure interferes slightly with reacquisition only when saccharin is the test solution makes no intuitive sense and is not supported by any other result in the present series of experiments. Furthermore, even in Figure 2, the statistical evidence for it is not strong and our opinion is that it is a sampling error. Although the result was significant at a conventional .05 level, it must be remembered that a 2 by 2 by 2 ANOVA yields seven $F$ ratios and hence there is a problem with experimentwise Type I error.

\section{EXPERIMENT 3}

Experiments 1 and 2 suggest that rats have no difficulty reacquiring an extinguished taste aversion. The contradiction between these findings and the report of Danguir and Nicolaides (1977) might have been due to procedural differences. Hence, we now report two replications of their Experiment 1, in which Group Exp was trained to have an aversion to un- 
flavored water, while Group Cont was not; after extinction of the aversion to unflavored water in Group Exp, both groups were subjected to reconditioning. Danguir and Nicolaides reported that Group Exp did not reacquire the aversion while Group Cont did.

\section{Method}

The range of weights for the 24 male Sprague-Dawley rats in Replication 1 was $229-245 \mathrm{~g}$ just prior to the experiment. The weight range for the 24 similar rats in Replication 2 was $360-400 \mathrm{~g}$. Each replication contained 12 rats in Group Exp and 12 rats in Group Cont.

\section{Procedure}

Phase 1: Adaptation ( 4 days in Replication 1 and 8 days in Replication 2). All rats were allowed $15 \mathrm{~min}$ daily access to unflavored water in steel cups. These cups were novel for the rats and, if a rat did not drink $5 \mathrm{~g}$ during the $15-\mathrm{min}$ session, it was administered additional sessions until it did so.

Phase 2: Conditioning and extinction (11 days). In Group Exp, $15 \mathrm{~min}$ of free access to unflavored water was allowed on each day. On the first 3 days of Phase 2, IP injections of $20 \mathrm{ml} / \mathrm{kg}$ of . $15 \mathrm{M}$ (.64\% weight/volume) $\mathrm{LiCl}$ solution were administered just as the cup was removed. On Days 5 and 6 of Phase 2, if a rat in Replication 1 did not drink $5 \mathrm{~g}$, the difference between $5 \mathrm{~g}$ and the amount it drank was placed in the cup and made available to the rat. Group Cont was allowed $15 \mathrm{~min}$ daily free access to water on Day 1 of Phase 2, but thereafter each rat in Group Cont was yoked with a different rat in Group Exp and rationed the same amount of water as the rat in Group Exp had consumed. In Replication $2,20 \mathrm{ml} / \mathrm{kg}$ of normal saline was used as a control for the lithium injection of Phase 2 in Group Cont; there was no such saline injection in Replication 1.

Phase 3: Reconditioning and testing (6 days). Both groups were allowed to drink unflavored water freely for $15 \mathrm{~min}$ per day. On Days 1 and 2 of Phase 3, all rats were injected with the $20 \mathrm{ml} / \mathrm{kg}$ $.15 \mathrm{M}$ lithium dose as the cups were removed.

Replication 2 differed explicitly from the corresponding experiment by Danguir and Nicolaides (1977) only in the following respects. All injections were administered immediately as the cup was removed, while Danguir and Nicolaides waited 5 min. Group Cont was not yoked with Group Exp for water consumption on Day 1 of Phase 2 and on Day 1 of Phase 3. There must also have been other differences with the Danguir-Nicolaides procedures, but we cannot specify them exactly. Our Sprague-Dawley Wistarderived rats probably differed genetically from the Wistars used by Danguir and Nicolaides, our tap water probably was slightly different, and there almost certainly were differences in the types of rat cages, water cups, and maintenance diets.

\section{Results}

All groups reacquired the aversion to unflavored water. Figure 3 shows mean water consumption for each group on the reconditioning days (Days 1 and 2 of Phase 3) and on subsequent test days separately for the two replications. Each of the four groups in Figure 3 drank less on Day 2 than on Day 1, which was the first reconditioning day; each group drank less on Day 3 than on Day 2, which was the second reconditioning day; each group drank more on Day 4 than on Day 3, which was the first extinction day (.05 level, paired $t$ tests). In the case of Group Exp of Replication 2, the reacquisition of the aversion

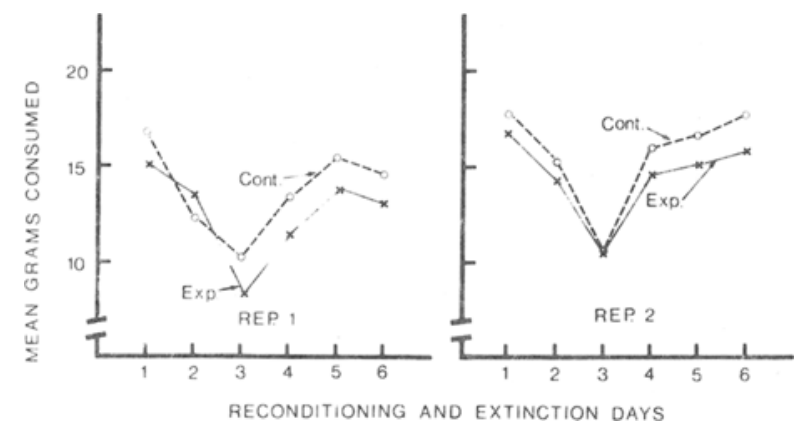

Figure 3. Acquisition and extinction of an aversion to unflavored water in Experiment 3. Group Exp had a prior history of acquisition and extinction of such an aversion while Group Cont did not.

after a single reconditioning trial and its intensification after a second reconditioning trial are in flat contradiction to the results reported by Danguir and Nicolaides (1977).

\section{EXPERIMENT 4}

In a second experiment, Danguir and Nicolaides (1977) found that rats with a history of extinction of a saccharin aversion would not reacquire it with reconditioning. Two control groups without such a history were later able to acquire a saccharin aversion. One control group received a saline injection instead of a lithium injection on the original conditioning day. A second control group received an injection of lithium $2 \mathrm{~h}$ prior to drinking saccharin solution; it is known that conditioning will not occur under such circumstances (Boland, 1973). Here we report two independent replications of this experiment.

\section{Method}

In unspecified details, the method was like that of Experiment 3.

The 36 rats in Replication 1 weighed $229-250 \mathrm{~g}$ ad lib just prior to the experiment and were habituated to the deprivation schedule by being given unflavored water in cups for $15 \mathrm{~min}$ per day for 4 days prior to the experiment. The 36 rats in Replication 2 weighed 321-358 $\mathrm{g}$ and received 1 day of preexperimental habituation. Each group in each replication contained 12 rats.

\section{Procedure}

Phase 1: Conditioning and extinction ( 9 days). Group Exp received $15 \mathrm{~min}$ daily of free access to $.1 \%(\mathrm{w} / \mathrm{v})$ sodium saccharin solution. On Day 1, this was followed immediately by $20 \mathrm{ml} / \mathrm{kg}$ of .15 M LiCl. Group Li-Cont received this Day $1 \mathrm{LiCl}$ injection $2 \mathrm{~h}$ prior to the saccharin drinking session. In Replication I, Group Sal-Cont received no injection on Day 1, while in Replication 2, this group received an injection of normal saline equal in volume to the lithium injection. On Days 2-9, the saccharin consumptions of the two control groups were yoked to Group Exp by the method used in Experiment 3.

Phase 2: Reconditioning and extinction (4 days). All rats had 15 min daily access to the $1 \%$ saccharin solution. On Day 1 of Phase 2, this was followed immediately by an injection of $20 \mathrm{ml} / \mathrm{kg}$ of $.15 \mathrm{M} \mathrm{LiCl}$.

The present procedure differed explicitly from the Danguir- 


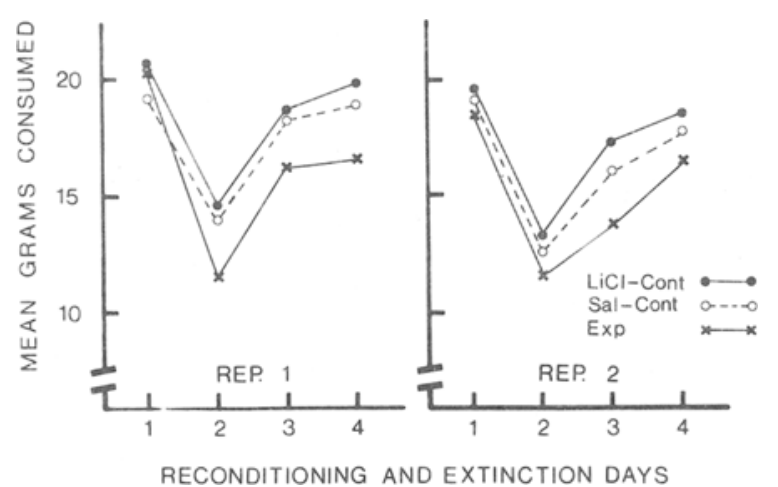

Figure 4. Acquisition and extinction of an aversion to saccharin solution in Experiment 4. Group Exp had a prior history of acquisition and extinction of such an aversion, while Groups LiCont and Sal-Cont did not.

Nicolaides procedure in that there was some habituation to the drinking schedule before the experiment proper, the injections were immediate, no control saline injection was administered to Group Sal-Cont in Replication 1, and the control rats were not yoked with the experimental rats on Days 1 and 10.

\section{Results}

Figure 4 shows the mean consumption of saccharin solution for each group on Day 1 of Phase 2, the reconditioning day, and on each subsequent extinction day. Each group in each replication drank less on Day 2 than on Day 1 in Figure 4, thus exhibiting conditioning, and each group drank more on Day 3 than on Day 2, thus exhibiting extinction (two-tail, .05-level paired t tests). A 2 by 3 ANOVA (Replications by Groups) for the total amount consumed during Days 2-4 in Figure 4 revealed a difference among groups $[F(2,66)=6.19, p<.005]$ as its only significant effect. Combined over both replications, Group Exp drank less than Group Li-Cont [t(66) = $3.41, \mathrm{p}<.005]$ or Group Sal-Cont $[\mathrm{t}(66)=1.92$, $\mathrm{p}<.10$, two tails]. This result suggests that prior extinction of a flavor aversion enhances reconditioning and is exactly opposite to that reported by Danguir and Nicolaides (1977). The two control groups did not differ significantly $[\mathrm{t}(66)=1.49]$.

\section{GENERAL DISCUSSION}

Taken together, the present experiments have yielded 10 statistically independent demonstrations that rats have no difficulty in reacquiring a previously extinguished learned taste aversion. In the case of two of these demonstrations in Experiment 2, there was some evidence that extinction of a learned saccharin aversion might interfere with later reacquisition, but in the case of two other demonstrations in Experiment 4, there was stronger evidence in the opposite direction. We do not know why Danguir and Nicolaides (1977) failed to recondition a previously extinguished flavor aversion, but we do think that their finding has little generality. There is no good reason to doubt the efficacy of booster chemical aversion treatments for human alcoholics. This is not to say that we are certain that the present results are universally applicable: There are a number of unpublished reports of failures of reacquisition and these suggest that we be cautious.

\section{REFERENCES}

Boland, F. Saccharin aversions induced by lithium chloride toxicosis in a backward conditioning paradigm. Animal Learning \& Behavior, 1973, 1, 3-4.

Boland, F., Mellor, C. S., \& Revusky, S. Chemical aversion treatment of alcoholism: Lithium as the aversive agent. Behaviour Research and Therapy, 1978, 16, 401-409.

DANGuir, J., \& Nicolaides, S. Lack of reacquisition in learned taste aversions. Animal Learning \& Behavior, 1977, 5, 395-397.

Elkins, R. L. Conditioned flavor aversions to familiar tap water in rats: An adjustment with implications for aversion therapy treatments of alcoholism and obesity. Journal of Abnormal Psychology, 1974, 83, 411-417.

Farley, J. A., Mclaurin, W. A., Scarborough, B. B., \& RAwlings, T. D. Pre-irradiation saçcharin habituation: A factor in avoidance behavior. Psychological Reports, 1964, 14, 491-496.

Lemere, F., \& Voegtrin, W. L. An evaluation of the aversion treatment for alcoholism. Quarterly Journal of Studies of Alcohol, 1950, 11, 199-204.

Nachman, M., \& Ashe, J. H. Learned taste aversions in rats as a function of concentration and route of administration of LiCl. Physiology \& Behavior, 1973, 10, 73-78.

Revusky, S. H. Some laboratory paradigms for the chemical aversion treatment of alsoholism. Journal of Behavior Therapy and Experimental Psychiatry, 1973, 4, 15-17.

REvUSKY, S., \& GoRry, T. Flavour aversions produced by contingent drug injection: Relative effectiveness of apomorphine, emetine, and lithium. Behaviour Research and Therapy, 1973, 11, 403-409.

Revusky, S., Parker, L. A., Coombes, J., \& Coombes, S. Rat data which suggest alcoholic beverages ought to be swallowed during chemical aversion therapy, not just tasted. Behaviour Research and Therapy, 1976, 14, 189-194.

REvusky, S., \& TAukulis, H. K. The effects of alcohol and lithium habituation on the development of alcohol aversions through contingent lithium injection. Behaviour Research and Therapy, 1975, 13, 163-166.

Revusky, S., Taukulis, H. K., Parker, L. A., \& Coombes, S. Chemical aversion therapy: Rat data suggest that it may be countertherapeutic to pair alcohol sedation with sickness. Behaviour Research and Therapy, 1979, 17, 177-188.

Voegtlin, W. L., Lemere, F., \& Broz, W. R. Conditioned reflex therapy of alcohol addiction III: An evaluation of present results in light of previous experience with the method. Quarterly Journal of Studies on Alcohol, 1940, 1, 501-515.

Wiens, A. N., Montague, J. R., Manaugh, T. S., \& English, G. J. Pharmacological aversive counterconditioning to alcohol in a private hospital: One-year follow-up. Journal of Studies on Alcohol, 1976, 37, 1320-1324.

(Received for publication August 17, 1978; revision accepted January $26,1979$. 